Supporting Information for

\title{
Comprehensive identification of Amadori compound-modified phosphatidylethanolamines in
} human plasma

Xiaobo He, ${ }^{1}$ Guan-Yuan Chen, ${ }^{1}$ Qibin Zhang ${ }^{1,2^{*}}$

1. Center for Translational Biomedical Research, University of North Carolina at Greensboro, North Carolina Research Campus, Kannapolis, NC 28081, USA

2. Department of Chemistry \& Biochemistry, University of North Carolina at Greensboro, Greensboro, NC 27412, USA

* Email Address: q zhang2@uncg.edu

\section{Table of Contents}

\section{Content}

Page

Table S1. Summary of metadata of human blood donors self-reported as healthy.

Table S2. List of Amadori-PE (Am-PE) and Amadori-LysoPE (Am-LPE) species identified from human plasma extract and from in vitro reaction of human plasma extract with D-glucose using LC-NLS-MS and LC-PIS-MS in tandem.

Table S3. Intra-day and inter-day precision and accuracy of the method for quantitation of AmadoriPE/LysoPE. . $\mathrm{S5}$

Table S4. Matrix effects and extraction efficiencies of the method for quantitation of AmadoriPE/LysoPE.

Table S5. Stability of Amadori-PE/LysoPE under various conditions. 
Table S1. Summary of metadata of human blood donors self-reported as healthy. Plasma sample used in this study were pooled from these individual donors.

\begin{tabular}{|c|c|c|c|c|}
\hline & \multicolumn{2}{|c|}{ Female } & \multicolumn{2}{c|}{ Male } \\
\hline Race & $\mathbf{n}$ & Age (Mean \pm SD) (yrs) & $\mathbf{n}$ & Age (Mean \pm SD) (yrs) \\
\hline Asian & & & 1 & 20 \\
\hline Black & 47 & $36.5 \pm 12.8$ & 56 & $42.0 \pm 13.7$ \\
\hline Caucasian & 15 & $38.1 \pm 12.5$ & 13 & $51.5 \pm 10.7$ \\
\hline Hispanic & 54 & $35.2 \pm 12.7$ & 69 & $39.1 \pm 16.6$ \\
\hline Other & 1 & 23 & & \\
\hline $\begin{array}{c}\text { Grand } \\
\text { Total }\end{array}$ & 117 & $36.0 \pm 12.6$ & 139 & $41.3 \pm 15.4$ \\
\hline \multicolumn{2}{c}{} \\
\hline
\end{tabular}


Table S2. List of Amadori-PE (Am-PE) and Amadori-LysoPE (Am-LPE) species identified from human plasma extract and from in vitro reaction of human plasma extract with D-glucose using LC-NLS-MS and LC-PIS-MS in tandem.

\begin{tabular}{|c|c|c|c|c|c|c|}
\hline ID Species & Class & $\begin{array}{c}\mathrm{Rt} \\
(\mathrm{min})\end{array}$ & $\begin{array}{l}\text { human plasma } \\
\text { + glucose } \\
\text { PBA SPE }^{1}\end{array}$ & $\begin{array}{l}\text { human plasma } \\
+ \text { glucose }^{2}\end{array}$ & $\begin{array}{l}\text { human plasma, } \\
\text { MeOH-AA, } \\
\text { + PBA SPE }\end{array}$ & $\begin{array}{c}\text { human plasma, } \\
\mathrm{MeOH}-\mathrm{AA}^{4}\end{array}$ \\
\hline Am-LPE(0:0/18:3) & Am-LPE & 2.05 & + & $\mathrm{NF}^{5}$ & $\mathrm{NF}$ & $\mathrm{NF}$ \\
\hline $\operatorname{Am}-\operatorname{LPE}(20: 5 / 0: 0)$ & Am-LPE & 2.18 & + & + & NF & NF \\
\hline Am-LPE(18:3/0:0) & Am-LPE & 2.21 & + & + & $\mathrm{NF}$ & NF \\
\hline $\operatorname{Am}-\operatorname{LPE}(0: 0 / 22: 6)$ & Am-LPE & 2.44 & + & + & + & NF \\
\hline $\operatorname{Am}-\operatorname{LPE}(16: 1 / 0: 0)$ & Am-LPE & 2.49 & + & NF & NF & NF \\
\hline $\operatorname{Am}-\operatorname{LPE}(0: 0 / 20: 4)$ & Am-LPE & 2.54 & + & + & + & + \\
\hline $\operatorname{Am}-\operatorname{LPE}(22: 6 / 0: 0)$ & Am-LPE & 2.55 & + & + & + & + \\
\hline Am-LPE(0:0/18:2) & Am-LPE & 2.6 & + & + & + & + \\
\hline $\operatorname{Am}-\operatorname{LPE}(20: 4 / 0: 0)$ & Am-LPE & 2.71 & + & + & + & + \\
\hline $\operatorname{Am}-\operatorname{LPE}(18: 2 / 0: 0)$ & Am-LPE & 2.79 & + & + & + & + \\
\hline $\operatorname{Am}-\operatorname{LPE}(0: 0 / 20: 3)$ & Am-LPE & 3.03 & + & + & $\mathrm{NF}$ & NF \\
\hline $\operatorname{Am}-\operatorname{LPE}(0: 0 / 16: 0)$ & Am-LPE & 3.23 & + & + & $\mathrm{NF}$ & + \\
\hline $\operatorname{Am}-\operatorname{LPE}(20: 3 / 0: 0)$ & Am-LPE & 3.25 & + & + & $\mathrm{NF}$ & NF \\
\hline Am-LPE(16:0/0:0) & Am-LPE & 3.51 & + & + & + & + \\
\hline $\operatorname{Am}-\operatorname{LPE}(0: 0 / 18: 1)$ & Am-LPE & 3.52 & + & + & $\mathrm{NF}$ & NF \\
\hline $\operatorname{Am}-\operatorname{LPE}(0: 0 / 22: 4)$ & Am-LPE & 3.58 & + & $\mathrm{NF}$ & $\mathrm{NF}$ & NF \\
\hline $\operatorname{Am}-\operatorname{LPE}(22: 4 / 0: 0)$ & Am-LPE & 3.8 & + & $\mathrm{NF}$ & $\mathrm{NF}$ & NF \\
\hline Am-LPE(18:1/0:0) & Am-LPE & 3.81 & + & + & + & + \\
\hline Am-LPE(0:0/18:0) & Am-LPE & 5 & + & + & + & + \\
\hline $\operatorname{Am}-\operatorname{LPE}(18: 0 / 0: 0)$ & Am-LPE & 5.45 & + & + & + & + \\
\hline Am-PE(18:2/20:4) & Am-PE & 11.76 & + & + & NF & NF \\
\hline Am-PE(16:0/20:5) & Am-PE & 11.83 & + & NF & NF & NF \\
\hline Am-PE(18:2/18:2) & Am-PE & 11.91 & + & + & $\mathrm{NF}$ & NF \\
\hline Am-PE(16:0/18:3) & Am-PE & 11.96 & + & NF & NF & NF \\
\hline Am-PE(16:0/22:6) & Am-PE & 12.51 & + & + & + & + \\
\hline Am-PE(18:1/22:6) & Am-PE & 12.6 & + & + & + & + \\
\hline Am-PE(16:0/20:4) & Am-PE & 12.78 & + & + & + & + \\
\hline Am-PE(16:0/18:2) & Am-PE & 12.93 & + & + & + & + \\
\hline $\operatorname{Am}-\operatorname{PE}(18: 1 / 20: 4)-1$ & Am-PE & 12.93 & + & + & + & + \\
\hline Am-PE(18:1/18:2) & Am-PE & 13.09 & + & + & + & + \\
\hline Am-PE(P-16:0/22:6) & Am-PE & 13.16 & + & + & + & + \\
\hline $\operatorname{Am}-\mathrm{PE}(18: 1 / 20: 4)-2$ & Am-PE & 13.18 & + & + & NF & NF \\
\hline Am-PE(P-18:1/22:6)-1 & Am-PE & 13.3 & + & + & + & + \\
\hline Am-PE(16:0/20:3) & Am-PE & 13.37 & + & + & + & NF \\
\hline Am-PE(O-16:0/22:6) & Am-PE & 13.42 & + & + & + & $\mathrm{NF}$ \\
\hline Am-PE(18:0/20:5) & Am-PE & 13.43 & + & + & + & + \\
\hline Am-PE(P-18:0/22:6)-1 & Am-PE & 13.5 & + & NF & NF & NF \\
\hline Am-PE(P-16:0/20:4) & Am-PE & 13.52 & + & + & + & + \\
\hline Am-PE(P-18:1/22:6)-2 & Am-PE & 13.54 & + & + & $\mathrm{NF}$ & NF \\
\hline Am-PE(P-18:1/20:4)-1 & Am-PE & 13.63 & + & + & + & + \\
\hline
\end{tabular}




\begin{tabular}{|c|c|c|c|c|c|c|}
\hline Am-PE(P-16:0/18:2) & Am-PE & 13.72 & + & + & + & + \\
\hline Am-PE(P-18:1/18:2) & Am-PE & 13.78 & + & + & + & + \\
\hline Am-PE(16:0/22:4) & Am-PE & 13.83 & + & $\mathrm{NF}$ & $\mathrm{NF}$ & NF \\
\hline Am-PE(O-16:0/22:5) & Am-PE & 13.86 & + & + & + & + \\
\hline Am-PE(P-18:1/20:4)-2 & Am-PE & 13.91 & + & + & + & NF \\
\hline Am-PE(O-16:0/18:2) & Am-PE & 13.95 & + & + & + & NF \\
\hline Am-PE(18:0/22:6) & Am-PE & 14.04 & + & + & + & + \\
\hline Am-PE(16:0/18:1) & Am-PE & 14.07 & + & + & + & + \\
\hline Am-PE(P-16:0/20:3) & Am-PE & 14.09 & + & $\mathrm{NF}$ & $\mathrm{NF}$ & + \\
\hline Am-PE(P-16:0/22:5) & Am-PE & 14.14 & + & + & + & NF \\
\hline Am-PE(18:1/18:1) & Am-PE & 14.22 & + & + & + & + \\
\hline Am-PE(18:0/20:4) & Am-PE & 14.35 & + & + & + & + \\
\hline $\operatorname{Am}-\mathrm{PE}(18: 0 / 22: 5)-1$ & Am-PE & 14.46 & + & + & + & + \\
\hline Am-PE(18:0/18:2) & Am-PE & 14.51 & + & + & + & + \\
\hline Am-PE(P-16:0/22:4) & Am-PE & 14.6 & + & $\mathrm{NF}$ & + & NF \\
\hline Am-PE(P-18:0/22:6)-2 & Am-PE & 14.74 & + & + & + & + \\
\hline Am-PE(O-16:0/22:4) & Am-PE & 14.82 & + & + & + & NF \\
\hline Am-PE(P-16:0/18:1) & Am-PE & 14.87 & + & + & + & + \\
\hline Am-PE(18:0/20:3) & Am-PE & 14.91 & + & + & + & + \\
\hline Am-PE(P-18:1/18:1) & Am-PE & 14.95 & + & + & + & + \\
\hline Am-PE(18:0/22:5)-2 & Am-PE & 14.95 & + & + & + & + \\
\hline Am-PE(O-16:0/18:1) & Am-PE & 14.95 & + & + & + & NF \\
\hline Am-PE(O-18:0/22:6) & Am-PE & 15.01 & + & + & + & + \\
\hline Am-PE(P-18:0/20:4) & Am-PE & 15.06 & + & + & + & + \\
\hline Am-PE(P-18:0/22:5)-1 & Am-PE & 15.16 & + & + & + & + \\
\hline Am-PE(P-18:0/18:2) & Am-PE & 15.25 & + & + & + & + \\
\hline Am-PE(O-18:0/20:4) & Am-PE & 15.32 & + & + & + & + \\
\hline Am-PE(18:0/22:4) & Am-PE & 15.35 & + & + & + & + \\
\hline Am-PE(16:0/18:0) & Am-PE & 15.42 & + & + & + & NF \\
\hline Am-PE(O-18:0/18:2) & Am-PE & 15.49 & + & + & + & NF \\
\hline Am-PE(18:0/18:1) & Am-PE & 15.56 & + & + & + & + \\
\hline Am-PE(P-18:0/22:5)-2 & Am-PE & 15.64 & + & + & + & + \\
\hline Am-PE(P-18:0/20:3) & Am-PE & 15.65 & + & + & + & + \\
\hline Am-PE(20:0/20:4) & Am-PE & 15.86 & + & $\mathrm{NF}$ & $\mathrm{NF}$ & NF \\
\hline Am-PE(P-18:0/22:4) & Am-PE & 16.01 & + & + & + & + \\
\hline Am-PE(P-18:0/18:1) & Am-PE & 16.23 & + & + & + & + \\
\hline Am-PE(O-20:0/20:4) & Am-PE & 16.24 & + & + & + & NF \\
\hline Am-PE(O-18:0/16:0) & Am-PE & 16.33 & + & + & $\mathrm{NF}$ & NF \\
\hline Am-PE(O-18:0/18:1) & Am-PE & 16.44 & + & + & + & + \\
\hline Am-PE(P-20:0/20:4) & Am-PE & 16.52 & + & + & + & + \\
\hline Am-PE(P-20:0/18:2) & Am-PE & 16.66 & + & + & + & + \\
\hline Am-PE(P-22:0/18:2) & Am-PE & 17.78 & + & + & + & NF \\
\hline
\end{tabular}


Table S3. Intra-day and inter-day precision and accuracy of the method for quantitation of AmadoriPE/LysoPE.

\begin{tabular}{cccc}
\hline & & $\begin{array}{c}\text { Amadori- } \\
\text { LysoPE }\end{array}$ & $\begin{array}{c}\text { Amadori- } \\
\text { PE }\end{array}$ \\
\hline Intra-day & LQC & 7.8 & 0.9 \\
precision (\%) & MQC & 6.2 & 3.0 \\
& HQC & 6.7 & 5.2 \\
Inter-day & LQC & 5.3 & 4.5 \\
precision (\%) & MQC & 4.4 & 3.7 \\
& HQC & 9.5 & 4.4 \\
\hline \multirow{2}{*}{ Intra-day } & LQC & 109.5 & 107.4 \\
accuracy (\%) & MQC & 97.9 & 98.8 \\
& HQC & 97.6 & 89.1 \\
Inter-day & LQC & 107.2 & 107.7 \\
accuracy (\%) & MQC & 97.5 & 96.0 \\
& HQC & 102.0 & 88.3 \\
\hline
\end{tabular}

Table S4. Matrix effects and extraction efficiencies of the method for quantitation of AmadoriPE/LysoPE.

\begin{tabular}{cccc}
\hline & & $\begin{array}{c}\text { Amadori- } \\
\text { LysoPE }\end{array}$ & $\begin{array}{c}\text { Amadori- } \\
\text { PE }\end{array}$ \\
\hline Matrix Effect & LQC & $86.0 \pm 3.3$ & $97.9 \pm 1.8$ \\
$($ ME\% \pm RSD) & HQC & $91.7 \pm 5.1$ & $94.0 \pm 7.2$ \\
& HQC & $89.8 \pm 7.5$ & $92.8 \pm 8.1$ \\
\hline Extraction & LQC & $57.5 \pm 4.2$ & $43.7 \pm 6.5$ \\
Efficiency & MQC & $57.4 \pm 8.3$ & $51.4 \pm 12.7$ \\
$(\mathrm{PE} \% \pm \mathrm{RSD})$ & HQC & $47.3 \pm 2.0$ & $39.1 \pm 8.0$ \\
\hline
\end{tabular}

Table S5. Stability of Amadori-PE/LysoPE under various conditions.

\begin{tabular}{llcc}
\hline & Conditions & $\begin{array}{c}\text { Amadori- } \\
\text { LysoPE }\end{array}$ & $\begin{array}{c}\text { Amadori- } \\
\text { PE }\end{array}$ \\
\hline \multirow{3}{*}{ Bench-top } & $\mathrm{RT}, 4 \mathrm{~h}$ & $93.4 \pm 5.3$ & $98.9 \pm 3.2$ \\
& $\mathrm{RT}, 12 \mathrm{~h}$ & $102.6 \pm 4.6$ & $103.7 \pm 3.5$ \\
& $\mathrm{RT}, 24 \mathrm{~h}$ & $82.3 \pm 9.0$ & $94.2 \pm 5.9$ \\
\hline Processed & $10^{\circ} \mathrm{C}, 4 \mathrm{~h}$ & $99.1 \pm 2.8$ & $95.4 \pm 1.9$ \\
sample & $10^{\circ} \mathrm{C}, 12 \mathrm{~h}$ & $104.7 \pm 5.3$ & $97.5 \pm 6.2$ \\
\hline $\begin{array}{l}\text { Freeze-and- } \\
\text { thaw }\end{array}$ & $10^{\circ} \mathrm{C}, 24 \mathrm{~h}$ & $95.8 \pm 8.1$ & $102.9 \pm 6.1$ \\
\hline
\end{tabular}

